\title{
Evaluating the effectiveness of virtual fracture clinics for ankle injuries
}

\author{
Andrew Robinson, Anand Pillai and Mohamad Alqubaisi* \\ University Hospital of South Manchester NHS Foundation Trust, UK
}

\begin{abstract}
Background and objectives: The virtual fracture clinic protocol was adopted at UHSM to reduce the number of unnecessary referrals to the outpatient fracture clinic. I will be reviewing literature and providing an audit report to evaluate whether the protocol is effective for managing ankle injuries.
\end{abstract}

Method: I collected data on every patient that was reviewed in the VFC with an ankle injury during a three-month period which was a sample population of 100 This data consisted of patient gender, age, mode of injury, VFC diagnosis, treatment, whether the patient had a follow-up and, if so, how many.

Results: Out of the 100 patients, 24 were discharged and 76 had follow-ups arranged. Out of the 76 patients, 3 had follow-ups at other hospitals and 2 cancelled theirs; these 5 patients were then excluded. Of the 71 patients who had follow-ups, 37 patients required 3 or more follow-ups.

Conclusion: I think the protocol is effective for treating patients with ankle injuries. $24 \%$ of patients were discharged following the VFC. Therefore, there was a significant reduction of unnecessary fracture clinic referrals for these patients. This allows the re-allocation of staff resources to deal with more complex injuries.

\section{Introduction}

Traditionally, patients with acute fractures present to their local Emergency Department (ED) and are referred to the Fracture Clinic unless an orthopaedic opinion is required. In which case a member of the orthopaedic team is sent to the ED to reassess the patient, it is usually the SHO or another junior member of the team [1].

The large number of referrals to orthopaedics causes many subsequent unnecessary clinics, even for patients with stable, nondisplaced fractures and the re-assessment of patients' fractures in the ED consumes the time of the orthopaedic staff as well as slowing the ED's patient flow [1].

One study state that through the use of virtual clinics, those working in the ED could safely discharge patients with stable fractures and reduce the number of fracture clinic referrals by more than $30 \%$. Increasing the amount of time orthopaedic clinicians have more complex injuries [2].

Therefore, at the University Hospital of South Manchester (UHSM), the orthopaedic department and the emergency department have decided to redesign their protocols used for fractures.

The new protocol consists of definitive management of many stable fractures purely in the ED with virtual fracture clinics to review patient notes and radiographs in order to make a decision for each patient to either arrange a follow-up clinic or to discharge them and continue with the treatment outlined by the ED where the doctors should be abiding by the agreed treatment guidelines. There is an emphasis placed on 'self-care' for patients that are discharged, and this is supported by informative leaflets on how to manage their specific injury.

I will use the audit data I have collected to evaluate the patient outcomes (efficacy) after the use of this new protocol for specifically ankle injuries, which I feel is the most important factor in deciding whether or not the new protocol is effective but I will also refer to other factors such as the cost-effectiveness of and the patient satisfaction in regard to virtual fracture clinics as a whole using various pieces of literature I have found on PubMed.

\section{Literature review}

\section{Search methods}

Initially I searched "(Virtual Fracture Clinic) AND (Ankle Injuries)” on PubMed however this only returned one result, this paper is relevant to my literature review but is unfortunately regarding only Paediatric injuries. And so, I tried searching (virtual fracture clinic malleolus) on google scholar, even though I am aware that these sources can be less reliable, which returned 455 results. Although I could only locate one article from this search of relevance to my review. Due to the lack of papers linking virtual fracture clinics with ankle injuries, I decided to evaluate literature about virtual fracture clinics in general.

In order to find more literature, I searched solely "(Virtual Fracture Clinic)" and published within the last 10 years on PubMed which yielded 18 results, most of the relevant virtual fracture clinic papers were published between 2014 and now showing it is quite a new concept. I used these results to begin my research.

\section{Efficacy}

The virtual fracture clinic allows patients to be reviewed quickly and effectively, this is displayed by an audit carried out at Leicester

${ }^{*}$ Correspondence to: Mohamad Alqubaisi, University Hospital of South Manchester NHS Foundation Trust, UK, E-mail: mohamad.alqubaisi@mft.nhs.uk

Received: September 24, 2018; Accepted: October 12, 2018; Published: October 16,2018 
Royal Infirmary. A total of 797 patients were processed through the protocol in 4 weeks, the average number of patients discussed in the virtual fracture clinic per day was 28 at an average of 1 minute per patient.

In contrast, the average time taken to review a patient in the routine out-patient fracture clinic was 11 minutes. Therefore, if the new protocol hadn't been introduced at this institution, the 28 patients per day would have taken 308 minutes instead of 28 minutes [3]. These results are represented in the table 1 below.

This table highlights the time that can be saved by using this new protocol and these staff resources could be allocated to other more complex injuries that require attention.

The main concern with the concept of the virtual fracture clinic is that patient's presenting to the ED may be discharged with a diagnosed stable fracture and no follow-up when, in fact, they have a potentially serious injury that was missed by a clinician.

However, one study was carried out to assess for flaws in the new protocol and one of its hypotheses was that if there was no routine orthopaedic follow-up, patients may need re-visit the ED with the same injury if it doesn't improve. The results show that the unplanned reattendances of fracture patients did not increase with the new protocol. Suggesting that the redesigned protocol has low likelihood of poor patient outcomes [4].

An example of a stable fracture is a 5th metacarpal fracture which represents $20 \%$ of all hand fractures. It is a fracture that can be managed definitively in the ED without the need for a formal orthopaedic follow-up [5].

A study tested out this protocol on 5th metacarpal fractures and the results stated that $80.6 \%$ of patients were either very satisfied or satisfied with the outcome of their injury, suggesting that for the majority of cases, the discharge from the ED with 'self-care' information and no formal follow-up with the fracture clinic is adequate treatment for patients with stable fractures [5].

Conventionally, patients have been recalled for early and mediumterm follow-ups in orthopaedic clinics to assess fracture position and functional recovery. However, the new protocol places emphasis on 'self-care' which is supported by verbal information from the ED and also written information in the form of leaflets.

This reduces the amount of unnecessary clinic follow-ups which has significant benefits to orthopaedic departments, which will have more staff resources available to devote to more challenging, complex injuries [6].

Another study, this time on whether or not follow-ups were necessary for 5 th metatarsal injuries concluded that there was no added clinical value of routine outpatient follow-ups for these fractures. These patients can be safely discharged after the ED and are allowed to bear weight starting immediately.

In this study, fracture clinic attendances and outcomes were audited for 1 year before and 1 year after the new protocol was introduced in 2011. There was a reduction in the number of follow-up clinics required

Table 1. Comparison of time taken to review patients virtually against formally [3]

\begin{tabular}{|l|c|c|}
\hline & Virtual Fracture Clinic & Outpatient Fracture Clinic \\
\hline Patients seen/ day & 28 & 22 \\
\hline Average time/ day & 28 & 244 \\
\hline Average time/ patient & 1 & 11 \\
\hline
\end{tabular}

by a ratio of 5.87 with the new protocol in place with similar outcomes for patients and their [7].

\section{Cost-effectiveness}

One of the studies I found on PubMed which was carried out at Glasgow Royal Infirmary (GRI) reported that Virtual Fracture Clinics are a safe and effective alternative to traditional fracture clinics and also that they can reduce costs due to less overall use of staff resources [8].

The system was primarily used to improve quality of patient care but the reduction in unnecessary clinics after orthopaedic trauma and the associated cost savings were an added bonus. Between 2009 and 2014, the total staffing costs of the orthopaedic outpatient department (OPD) at GRI rose by $4 \%$ which is considerably less than the Scottish national increase by $16 \%$ [8].

The OPD's rate of attendance also fell by $15 \%$ compared to the national average which fell by $5 \%$. If GRI's attendance rates were the same as the national average, $£ 212,705$ would have been required to pay the excess expenditure. Extrapolation of this same data suggests that national adoption of this system may have the potential to achieve cost savings of $£ 3,535,808$ for the NHS [8]. I have displayed some of their data below in table 2 .

I chose this study to include in my review because it was based on national data collected over 6 years which shows the results are reliable and also because during this time period, there were no new protocols introduced in the department, other than the fracture pathway redesign. Therefore, the differences discovered in cost between GRI and the national average are likely to be as a result of this change.

\section{Patient satisfaction}

There is a good evidence base to show that conservative treatment with simple 'neighbour-strapping' and no follow-up leads to high patient satisfaction because the patients are able to get back to work and also it saves them arranging transport to the hospital for their follow-up clinics [5].

In a study conducted on patients with 5th metacarpal injuries that were treated with the new protocol, $84.9 \%$ reported being satisfied with the new process suggesting that the patients appreciate the conservative treatment and the lack of a requirement to travel to and from hospital for clinics [5].

A study was carried out to assess patient satisfaction concerning this fracture pathway redesign for patients with radial head and neck fractures, $77 \%$ of the patients responded to the survey about their experience with the process and the overall satisfaction was $96 \%$ in the suspected fracture group and $87 \%$ in the definite fracture group. These high satisfaction rates display that the patients are content with the treatment provided even with the limited face-to-face follow-ups [6] Below are the results of the survey in table 3.

This table displays the low rates of patient dissatisfaction with the new protocol which displays that only the low minority of patients are unhappy with how they were managed. It would be interesting to investigate why they were not happy with the treatment, possibly due to a lack of information leaving the patient unable to self-manage their injury or they might have had a further complication of their injury.

\section{In summary}

All of the literature that I found on PubMed was in support of the fracture clinic redesign, the message that I ascertained from a 
Table 2. Total staff costs from the OPD at GRI compared with the national average. Percentages represent change from the 2009 baseline [8]

\begin{tabular}{|c|c|c|c|c|}
\hline Year & $\begin{array}{c}\text { Study } \\
\text { Institution }\end{array}$ & & $\begin{array}{l}\text { National } \\
\text { Average }\end{array}$ & \\
\hline & Staff Cost (£) & $\%$ Change & Staff Cost (£) & $\%$ Change \\
\hline 2009 & $1,744,933$ & & $29,213,145$ & \\
\hline 2010 & $1,664,126$ & -5 & $30,686,629$ & 5 \\
\hline 2011 & $1,733,230$ & -1 & $32,508,444$ & 11 \\
\hline 2012 & $1,930,048$ & 11 & $35,513,636$ & 22 \\
\hline 2013 & $1,845,820$ & 6 & $33,663,199$ & 15 \\
\hline 2014 & $1,811,301$ & 4 & $33,861,804$ & 16 \\
\hline
\end{tabular}

Table 3. Results of satisfaction survey [6]

\begin{tabular}{|c|c|c|c|c|}
\hline Satisfaction & Very satisfied & Satisfied & Unsatisfied & $\begin{array}{c}\text { Very } \\
\text { Unsatisfied }\end{array}$ \\
\hline $\begin{array}{c}\text { Suspected } \\
\text { fracture } \\
\text { Total (n=76) }\end{array}$ & $50 \%$ & $46 \%$ & $3 \%$ & $1 \%$ \\
\hline $\begin{array}{c}\text { Definite fracture } \\
\text { Total (n=79) }\end{array}$ & $29 \%$ & $58 \%$ & $9 \%$ & $4 \%$ \\
\hline
\end{tabular}

combination of inputs was that overall, the new treatment protocol is safe, has good clinical outcomes, high patient satisfaction and would be cost saving if deployed by the NHS.

I think it would be useful to quantify the clinical outcomes of the patients who pass through the new fracture clinic redesign using an orthopaedic score. I have used this study below as an example:

Eg. One retrospective study that was carried out in 2005 contacted every patient who presented to their ED with a mallet finger who had a minimum of a 6-month follow-up and assessed patient outcomes using the QuickDASH score.

QuickDASH is a shortened version of the full, thirty-item DASH Outcome Measure which is a reliable and valid measure of physical function and symptoms related to the upper-limb musculoskeletal disorders [9].

The median QuickDASH score was 2.3 which is a low median score, this suggests that the patient's all had good clinical outcomes because they are, in general, not experiencing much pain or functional debilitation from their injury. In which case, the conservative treatment was successful [10].

It would be possible to quantify the clinical outcomes after ankle injuries for patients who have been through the new protocol using the FADI score.

\section{Audit report}

\section{Background information}

A number of studies have evaluated the new virtual fracture clinic protocol and their results suggest that it could significantly reduce the number of unnecessary orthopaedic referrals with good patient outcomes.

This audit is assessing the efficacy of the virtual fracture clinic protocol for managing ankle injuries because this potentially costeffective protocol already shows evidence of good patient clinical outcomes for other fractures $[6,7]$.

\section{Method}

I have collected data over the past 4 weeks with a focus on specifically ankle injuries that have been reviewed in the virtual fracture clinic (VFC) at UHSM.
I have selected a three-month period ranging from the 20th October 2015 until the 21st January 2016 and listed the details of every ankle injury that was reviewed in the VFC within those dates. This formed a sample of 100 patients.

The data collected includes the patient's gender, age, VFC date, mode of injury, VFC diagnosis, treatment given, whether or not they were discharged from the VFC and if they weren't, the amount of face-toface follow-up appointments each patient received in the fracture clinic.

I will analyse and interpret the data in order to evaluate whether or not VFCs, as well as this new treatment protocol, are a suitable and effective method to implement in the management of the types of ankle fractures included in the data I have collected.

Before I begin the analysis, I would like to emphasise that, due to the small sample size of only 100 patients, it will be hard to form accurate deductions and interpretations from the data collected about the less prevalent injuries that have a low incidence within the sample used for this report. It is also possible due to the small sample size that the results and trends seen in this report are not a true reflection of the local population.

Another further limitation to this audit report is the fact that not all types of ankle factures are included in the sample used, this disenables us from investigating whether the VFC protocol is suitable or effective for managing said types of ankle fractures.

\section{Results}

From the sample of 100 patients with ankle injuries reviewed in the VFC, $76 \%$ had face-to-face follow-ups arranged at the orthopaedic outpatient clinic and the remaining $24 \%$ were discharged following their review.

Table 4 summarises the top five injuries in order of incidence for the injuries that were discharged following VFC and the injuries that required follow-up(s):

The main inconsistencies of VFC outcome were found in the following injuries:

- Distal Fibular Fractures- 6 were discharged, 10 had follow-ups.

- Weber A Fractures-3 were discharged, 7 had follow-ups.

- Soft tissue injuries-11 was discharged, 3 had follow-ups. (2 out of 3 follow-ups were only required to remove a backslab)

Of the 76 patients that required follow-ups, 3 had follow ups at other hospitals and 2 cancelled their follow-up appointments. This leaves 71 patients who all had at least one follow-up at UHSM's fracture clinic. These 71 patients collectively had 184 follow-ups.

Table 5 illustrates the number of patients who have had 1, 2, 3, 4, or 5+ follow-ups:

The patient sample consisted of 42 males and 58 females. I noticed that of the 42 males, 8 were discharged with the remaining 34 requiring follow-ups, meaning $19 \%$ of males were discharged after the VFC

In comparison, the females had a higher percentage of discharge. Of the 58 females reviewed in the VFC, 16 were discharged and 42 returned for follow-ups. And so, $28 \%$ of females were discharged following the VFC. Table 6 clearly displays these figures:

I categorised the patients by age into four groups- minors $(<18$ years), young adults (18-40 years), mature adults ( $41-65$ years) and the elderly ( $>65$ years). 
Table 4. Top 5 most frequent injuries that were discharged from VFC or that had follow-ups

\begin{tabular}{|l|l|}
\hline Injuries that were discharged (24\%) & Injuries that required a follow-up(s) (76\%) \\
\hline 11 Soft Tissue Injuries & 16 Weber B Fractures \\
\hline 6 Distal Fibula Fractures & 12 Lateral Malleolar Fractures \\
\hline 3 Weber A Fractures & 10 Distal Fibular Fractures \\
\hline 2 Salter Harris type 1 Fracture & 10 Achilles Tear Injuries \\
\hline $\begin{array}{l}\text { 1 Medial Malleolar Fracture } \\
=1 \text { Talus Fracture }\end{array}$ & 7 Weber A Fractures \\
\hline
\end{tabular}

Table 5. The number of patients who have had 1,2,3,4, or 5+ follow-ups and the percentage over the total number of patients receiving follow-ups

\begin{tabular}{|c|c|c|}
\hline No. of Follow-ups & No. of patients & $\begin{array}{c}\text { Percentage of Patients who } \\
\text { had Follow-ups }\end{array}$ \\
\hline 1 & 12 & $17 \%$ \\
\hline 2 & 22 & $31 \%$ \\
\hline 3 & 25 & $35 \%$ \\
\hline 4 & 9 & $13 \%$ \\
\hline $5+$ & 3 & $4 \%$ \\
\hline
\end{tabular}

Table 6. Gender comparison between amount of discharges and follow-ups

\begin{tabular}{|l|c|c|}
\hline Gender & Male patients & Female Patients \\
\hline $\begin{array}{l}\text { Total Number reviewed in } \\
\text { VFC }\end{array}$ & 42 & 58 \\
\hline Discharged following VFC & 8 & 16 \\
\hline $\begin{array}{l}\text { Follow-up arranged } \\
\text { following VFC }\end{array}$ & 34 & 42 \\
\hline $\begin{array}{l}\text { Percentage of Patients } \\
\text { discharged following VFC }\end{array}$ & $19 \%$ & $28 \%$ \\
\hline
\end{tabular}

Table 7. the number of patients from each age category who were reviewed, discharged or followed up and percentage of discharged patients in each category

\begin{tabular}{|l|c|c|c|c|}
\hline Age Category & $\begin{array}{c}\text { Minor } \\
\mathbf{1 8 y r s}\end{array}$ & $\begin{array}{c}\text { Young adult } \\
\mathbf{1 8 - 4 0 y r s}\end{array}$ & $\begin{array}{c}\text { Mature adult } \\
\mathbf{4 1 - 6 5 y r s}\end{array}$ & $\begin{array}{c}\text { Elderly } \\
\mathbf{7 6 5 y r s}\end{array}$ \\
\hline Total Number Reviewed in VFC & 17 & 32 & 31 & 19 \\
\hline Discharged following VFC & 7 & 6 & 5 & 6 \\
\hline $\begin{array}{l}\text { Follow-up arranged following } \\
\text { VFC }\end{array}$ & 10 & 26 & 26 & 13 \\
\hline $\begin{array}{l}\text { Percentage of Patients } \\
\text { discharged following VFC }\end{array}$ & $41 \%$ & $19 \%$ & $16 \%$ & $32 \%$ \\
\hline
\end{tabular}

Table 7 below summarises the amount of each age category who were discharged following VFC, who had follow-ups and the percentage of patients that were discharged from each age group.

\section{Discussion}

During this discussion, I aim to work systematically through all the information that can be found in the 'RESULTS' section of this report and add any further analysis and interpretation of the trends highlighted there while also linking these points with concepts included in the literature review section.

$76 \%$ of the patients in the sample were recalled for a follow-up, this suggests that most ankle injuries that present to the VFC will require at least one follow-up appointment.

This is a very high rate of follow-ups when compared to some injuries referred to in my literature review such as the $5^{\text {th }}$ metatarsal injuries or $5^{\text {th }}$ metacarpal injuries. The journals I found assessing these injuries claimed that no routine follow-up is necessary [5,7].

Although the rate of follow-up is quite high, the remaining $24 \%$ of patients that were discharged following the VFC will not receive any face-to-face review or follow-up and this enables the re-allocation of staff resources to deal with more challenging injuries.
As mentioned in my lit review, the traditional outpatient fracture clinics are much more time-consuming than the VFCs involved in the new treatment protocol. One of the studies discovered that when using VFCs, the average time taken to review a patient was 1 minute, whereas the average time taken to review a patient face-to-face at the outpatient fracture clinic was 11 minutes. Suggesting that the new protocol is 11 times more time-efficient [3].

I noticed an inconsistency among the VFC outcomes about certain types of ankle injuries including mainly distal fibular fractures, Weber A fractures and soft tissue injuries. For example, 6 patients with distal fibular fractures were discharged following VFC but 10 patients with distal fibular fractures had follow-up clinics arranged. This is possibly due to an alternative or more traditional treatment protocol being implemented rather than abiding by the recommended guidelines agreed for the new treatment protocol. Otherwise the VFC outcome may vary depending on the severity of the fracture or damage for the injuries mentioned.

Of the 76 patients that required follow-up, 3 had follow-ups at other hospitals and 2 patients cancelled their arranged follow-up. These cancellations may be due to the patient's beliefs that their injury is improving and that they don't require a follow-up. Or perhaps they received a second opinion which convinced them that they didn't require a follow-up. It would be interesting to find out why these patients cancelled their appointments.

As for the remaining 71 patients who all did have at least one followup appointment at UHSM's fracture clinic for their ankle injury, they collectively had 184 follow-up appointments with 37 patients having more than 3 follow-ups each.

This displays that the majority of patients with ankle injuries will require face-to-face follow-up clinics to assess their injuries following VFC, but this does not necessarily mean that the fracture clinic redesign is not a suitable for the management of ankle injuries.

The 24 patients who were discharged following VFC represent patients that traditionally would have all been referred to fracture clinic but were filtered out by the new protocol.

If the average time taken for a VFC patient review is 10 minutes shorter than the average time taken to carry out a traditional review in an outpatient clinic then those 24 patients represent 4 hours that a clinician does not need to spend assessing patients [3].

I also found that females had a higher percentage of discharge when their ankle injuries are reviewed in the VFC than males. This trend may be due to skewed data from the small sample size or perhaps because females have stronger health-seeking behaviours than males which could cause them to present with less serious injuries.

In regard to the age categories, I was surprised by the lower incidence of injuries for the minors (17) and the elderly (19) when the incidence among young adults and mature adults was 32 and 31 respectively. I assumed minors and the elderly would be more likely to present with fractures because minors have soft, growing bones and the elderly have the degenerative changes of aging weakening theirs. The higher incidence with young and mature adults may be a reflection of the age distribution in the local area.

The elderly and minors also had a higher rate of discharge following VFC than young and mature adults which I did not expect either, this could potentially be a reflection of which age groups have stronger health-seeking behaviours. 


\section{Evaluation}

I think the methods used in this audit report had a lot of potential but due to the time-constraints, the sample population is too small. This is the main limitation of this report; the small sample population makes it difficult to decide if the trends seen in the data are an accurate representation of the trends in the local population.

Another limitation of the report is that the sample was taken too recently. Out of the 71 patients who had at least 1 follow-up at UHSM, 16 patients are still to be discharged which means there could have potentially been a lot more follow-ups.

Although finding out whether the patients required follow-ups and how many they had is a useful tool in deciding how suitable the VFC is for ankle injuries, I think that the clinical outcome and patient satisfaction are two very important aspects which require more investigation.

\section{Conclusion}

Ultimately, the literature I found is very supportive of the new virtual fracture clinic protocol, and provides an evidence base that displays how this protocol can be cost-effective, time-efficient, have good clinical outcomes and receive high patient satisfaction rates.

I feel that this system is most effective for stable fractures that can be discharged at the VFC without any follow-up. Although most of the ankle injuries still required a follow-up, nearly one quarter of them did not and I think the new protocol will aid the reduction of orthopaedic referrals and in turn produce cost savings and allow re-allocation of staff resources.

I would recommend that future studies carry out full assessments of a VFC including factors such as, cost-effectiveness, efficacy and patient satisfaction with a larger sample population to gain an insight into the overall picture of virtual fracture clinics and whether they are effective in managing all stable fractures.

\section{References}

1. Vardy J, Jenkins PJ, Clark K, Chekroud M, Begbie K, et al. (2014) Effect of a redesigned fracture management pathway and 'virtual' fracture clinic on ED performance. $B M J$ Open 4: e005282. [Crossref]

2. Ramasubbu B, Caffrey R, Namara R Mc, Deiratany S, Okafor I (2014) An evaluation of fracture clinic referrals in Ireland's busiest Paediatric Emergency Department. Arch Dis Child 99: A10.

3. Beiri A, Alani A, Ibrahim T, Taylor GJ (2006) Trauma rapid review process: efficient out-patient fracture management. Ann R Coll Surg Engl 88: 408-411. [Crossref]

4. Vardy J, Rymaszewski L, Begbie K, Anthony I, Chekroud M, et al. (2013) Fracture pathway redesign improves emergency department efficiency. Emerg Med J 30: 876.

5. Gamble D, Jenkins PJ, Edge MJ, Gilmour A, Anthony IC, et al. (2015) Satisfaction and functional outcome with "self-care" for the management of fifth metacarpal fractures. Hand (N Y) 10: 607-612. [Crossref]

6. Jayaram PR, Bhattacharyya R, Jenkins PJ, Anthony I, Rymaszewski LA (2014) A new "virtual" patient pathway for the management of radial head and neck fractures. $J$ Shoulder Elbow Surg 23: 297-301. [Crossref]

7. Ferguson KB, McGlynn J, Jenkins P, Madeley NJ, Kumar CS, et al. (2015) Fifth metatarsal fractures - Is routine follow-up necessary? Injury 46: 1664-1668. [Crossref]

8. Jenkins PJ, Morton A, Anderson G, Van Der Meer RB, Rymaszewski LA, et al. (2016) Fracture clinic redesign reduces the cost of outpatient orthopaedic trauma care. Bone Joint Res 5: 33-36. [Crossref]

9. Beaton DE1, Wright JG, Katz JN, Upper Extremity Collaborative Group (2005) Development of the QuickDASH: comparison of three item-reduction approaches. $J$ Bone Joint Surg Am 87: 1038-1046. [Crossref]

10. Brooksbank K, Jenkins PJ, Anthony IC, Gilmour A, Nugent MP, et al. (2014) Functional outcome and satisfaction with a "self-care" protocol for the management of mallet finger injuries: a case-series. J Trauma Manag Outcomes 8: 21. [Crossref]

Copyright: (C2018 Robinson A. This is an open-access article distributed under the terms of the Creative Commons Attribution License, which permits unrestricted use, distribution, and reproduction in any medium, provided the original author and source are credited. 